\title{
In Silico Antituberculosis Drug Designing using UCSF Chimera
}

\author{
Shabana Urooj, Advaita Dhariwal, Vandana Singh, Fadwa M. Alrowais
}

\begin{abstract}
For the humans' well-being, Mycobacterium Tuberculosis (MTB) is a fatal and adversary disease since years because of if its multidrug straining. MTB consumes nitrate as a substitute during breathing mechanism due to malingering of oxygen, therefore it increases the chances of survival. The nitrate/nitrite response (NarL) is a transcriptional governing protein. It is a two-constituent signal alteration mechanism used to stabilize nitrate enzyme that promote chemical drop and plan dehydrogenation. In this work, molecular docking using in-silico technique by benzofuran and naphthofuran byproducts has been performed. In-silico interaction of phosphodonors to NarL has been done. From the simulation results it is noticed that all compounds are binding to active site, therefore it is concluded that all benzofuran and naphthofuran byproducts partake on the dynamic site of NarL and are able to perform as leading molecule. To obtain results, SwissDock, UCSF Chimera and Protein-ligand Docking is majorly utilized.
\end{abstract}

Keywords: docking, NaRL Protein, phosphodonors, protein-ligand, SwissDOCK, UCSF Chimera.

\section{INTRODUCTION}

Tuberculosis (TB) is one amongst the ten major reasons of deaths globally. In 2018, 10 million TB cases were reported, out of which 1.6 million people deceased. In these 1.5 million, 0.3 million people were with Human Immunodefiency Virus (HIV). TB is a foremost destroyer of HIV positive patients. In the year 2018, a million of children suffered with TB and 2,51,000 collapsed. Multidrug-resistant TB (MDR-TB) is still a calamity for the mankind. According to the latest report of World Health Organization [1], $5,58,000$ new cases resistant to rifampicin were reported. Rifampicin is the supreme effective major medication. $82 \%$ of 5,58,000 patients were suffering from MDR-TB. Worldwide, every year the occurrence of TB is dropping with a rate of approximately 2\%. To reach meet up with 2020 milestones under End TB Strategy the falling rate per year need to be 4 to $5 \%$. A predictable 54 million survived against TB during 2000 to 2018. As per the healthy world targets and sustainable growth, TB should die out by the end of 2035

Revised Manuscript Received on December 30, 2019.

* Correspondence Author

Shabana Urooj*, Department of Electrical Engineering, College of Engineering, Princess Nourah Bint Abdulrahman University, Riyadh, Saudi Arabia. E-mail: SMUrooj@pnu.edu.sa, shabanaurooj@ieee.org

Advaita Dhariwal, Department of Electrical Engineering, School of Engineering Gautam Buddha University, Greater Noida (UP) India.

Vandana Singh, Department of Chemistry, School of Vocational Studies \& Applied Sciences, Gautam Buddha University, Greater Noida (UP) India.

Fadwa M. Alrowais Department of Computer Sciences, College of Engineering, Princess Nourah Bint Abdulrahman University, Riyadh, Saudi Arabia.

(C) The Authors. Published by Blue Eyes Intelligence Engineering and Sciences Publication (BEIESP). This is an open access article under the CC BY-NC-ND license (http://creativecommons.org/licenses/by-nc-nd/4.0/)
As per the strategic planning, following three measures are required to be placed widespread for effectual termination; $i$. unified patient-centered care and prevention, ii. intrepid policies and support systems, iii. enhances research and inventive work.

Accomplishments of the strategy will be subjected to the country's initiatives and implementations towards the vital ideologies viz. government liabilities and assessment, robust partnership with civilization bodies and organizations, safety and advancement of human privileges, adoption of the strategic goals with handshakes at country level.

The objective of tuberculosis drug innovation and growth is to attain TB drugs and drug cures that are well agreed and greater to those obtainable today in their worth, speed of action, safety and acceptability, comfort of use for all patient inhabitants, and availability.

\section{Drug Design}

Frequently mentioned as rational drug design created by the knowledge of a biotic goal. Drug is an utmost usual organic minor that triggers or impedes the role of a bio molecule viz. protein. Proteins in turn outcomes as a healing aid for patient. It includes molecule morphology in terms of its shape and charge. Drug design often essentially depend on modelling techniques. Computer based simulations makes the drug design and related discoveries more robust and convenient. Such kind of modelling techniques are called as computer aided drug design. Drug-design methods which reliance upon the proficiency of the three-dimensional structure of the target biomolecule are called as structure-based drug design. Moreover, tiny biopharmaceutical molecules as well as peptides and specifically therapeutic antibodies are increasingly significant category of drugs under the analysis of computational methods. The computational methods are exclusively contributing in improvisation in terms of similarity, selectivity, and solidity. MTB to the main drugs has shown the necessity for the expansion of novel and effective drugs for better-quality TB control [2]. Based on these facts, this work aimed to use combined in silico techniques for the discovery of potential inhibitors.

\section{MATERIALS AND METHODS}

In this work freely available web services, namely; SwissDock, UCSF Chimera and Protein-ligand Docking are used. The Structure of NarL: In the asymmetric unit this protein is a homotetramer, which contains four similar sub-units without covalently bound. They have self-governing lively site for each subunit namely; A, B, C, and $\mathrm{D}$ respectively. This assembly characterizes a $\mathrm{N}$-terminal type signal receiving area which contains four matching chains. 
Protein Data Bank has been utilized to obtain Mycobacterium tuberculosis - PDB ID: 3EUL. Crystal structure coordinates at $1.9^{\circ} \mathrm{A}$ signal receiving domain is supposed to be NarL regulator. In Silico antitubercular action investigation of benzofuran and naphthofuran is performed in this study. A derivatives-based analysis for the benzofuran and naphthofuran is reported in [3].

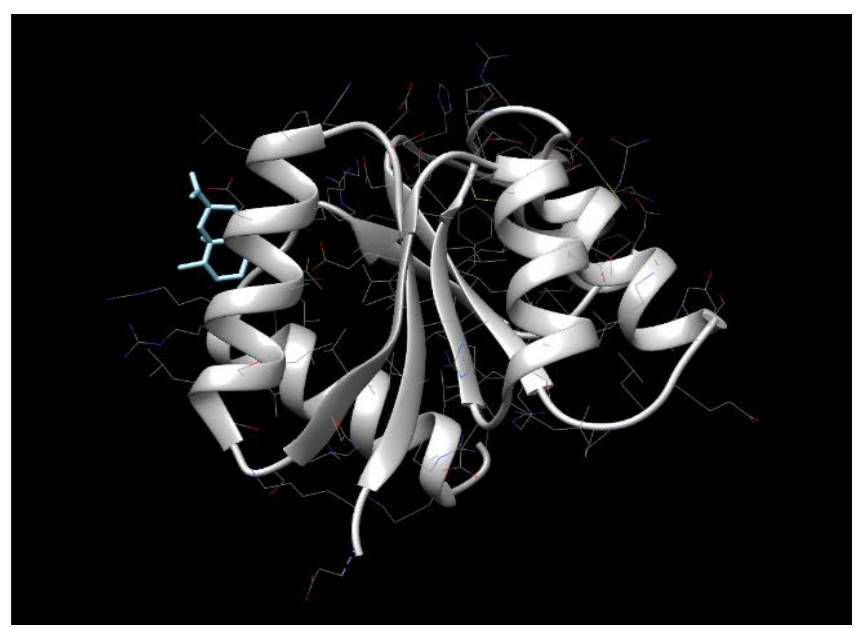

Fig. 1

The analysis of crystal structure of NarL protein displays exchanges between effector domains of complete regulator of bacterial response.

1. SwissDock: It is a protein ligand docking software which is freely available and it is based on EADock DSS software. This software is powered by research group of Swiss Institute of Bioinformatics. This free of cost web-service can visualize the molecular interactions which occurs between a tiny molecule and the large targeted protein. It can compute the complete morphology and characteristics viz. geometry, site and the energy of the peptides and small molecules which interacts with proteins.

2.UCSF Chimera: This noncommercial free web resource is developed by University of California San Francisco that is why it is abbreviated as UCSF. The UCSF Chimera often also called as Chimera. It is a package which is designed to make changes and allow addition of new abilities for its effective functioning. It is facility for interactive visualization and analysis of molecular structures. It can also be used to analyze the data related to the results obtained by docking and their trajectories. The predictions of SwissDock can be visualized by Chimera. SwissDock; an user-friendly online server targets an extensive technical research group for the docking of state-of-the-art protein, small molecules [4].

3. Protein-ligand Docking: This is a very wide procedure involved in the inventions and development of drugs. Molecular modelling is basis for analyzing the molecular interactions. The goal of this molecular modelling is to predict the morphology of a ligand; the tiny molecule during its bounding with a protein receptor or enzyme. It can visualize the orientation and positions of molecules, proteins and enzymes which is required for drug discovery and pharmaceuticals growth. Therapeutic research engages docking practices for diverse purposes; noticeably in virtual screening of huge databases available for chemicals for appropriate subject. Software used for the same purpose is 'SwissDock', which is able to compute geometry, site and energy of tiny molecules or peptides which intermingles with proteins.

\section{Process of Molecular Docking:}

1. By using the online server RCSB Protein Data Bank (PDB), the force field parameters and topology file has been generated for ligand.

2. Afterwards, the 'Target' controls have been added with protein atoms and the ligand. then partial charges were allocated to the ligand by SwissDock.

3. Protein and ligand docked together by using SwissDock.

4. Results from SwissDock were then analyzed by using UCSF Chimera.

Protein ligand interaction of: Acetyl phosphate (PDB ID: 4MVJ), Carbamoyl phosphate (PDB ID: 1CS0), Dihydroxyacetone-P (PDB ID: 3MHG), Phosphoramidate (PDB ID: 1TLP), Monophosphoimidazole (PDB ID: 1DS4), with protein target NarL (PDB ID: 3EUL) of MBT has been done to identify the antitubercular action.

\section{RESULTS AND DISCUSSIONS}

1. The in-silico interaction of phosphodonors with respect to NarL has been performed. It is detected that all the compounds were binding to active site.

2. It is also observed that all the natural phosphodonors muddled with active site. The energy ranges from -17.85 to $-25.704 \mathrm{kcal} / \mathrm{mol}$.

3. Out of 5 docked compounds, C5 demonstrates minimum binding energy, which is $-25.704 \mathrm{kcal} / \mathrm{mol}$ and $\mathrm{C} 4$ displays maximum binding energy $-17.85 \mathrm{kcal} / \mathrm{mol}$.

4. It is clear from the simulation results of molecular dynamics simulation for the study of compounds that all the derivatives of benzofuran and naphthofuran are in adequate vicinity to intermingle at NarL's active site. Moreover, they are capable to behave like main molecules.

\section{DOCKED RESULTS:}

1. Docking of Acetyl Phosphate with NarL

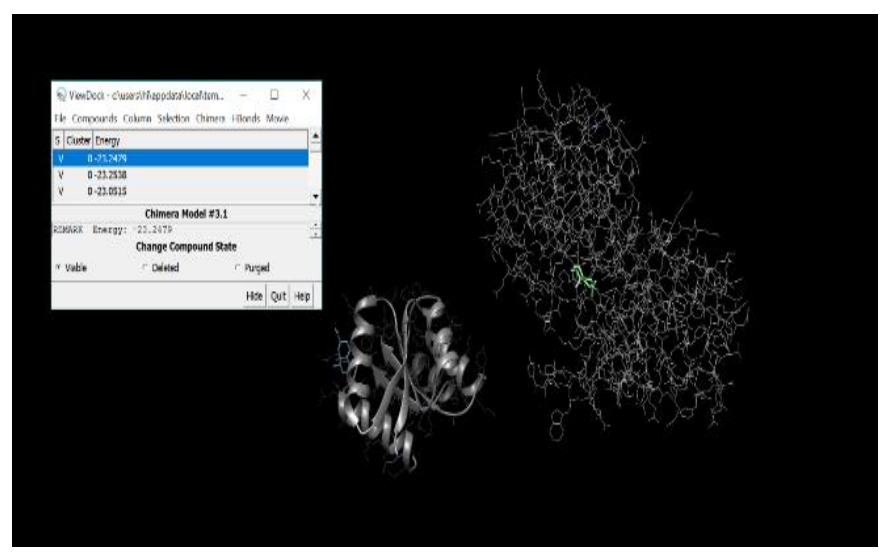

Fig.2. Docking of Acetyl Phosphate with NarL 


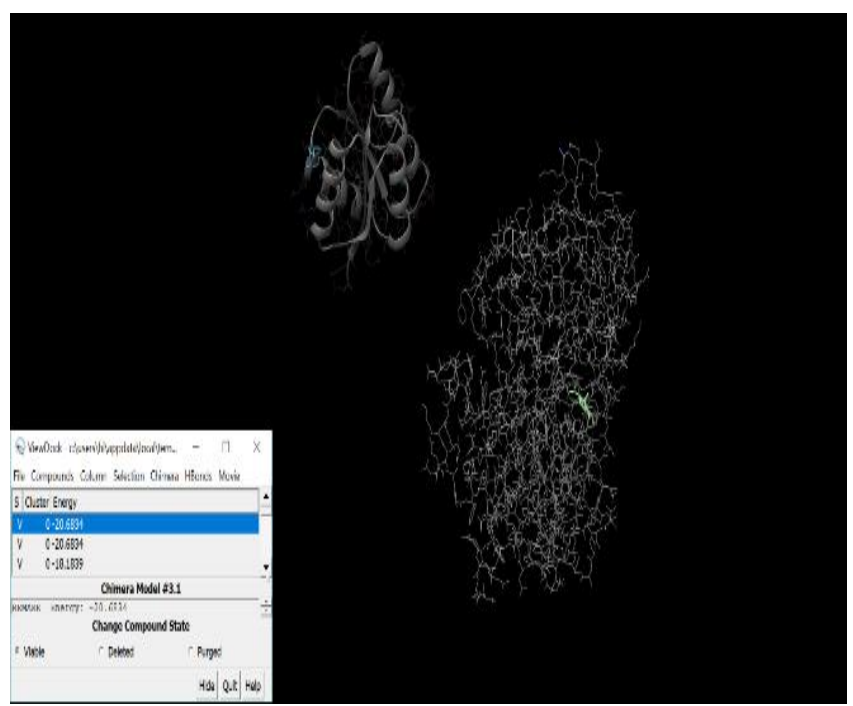

Fig.3. Docking of Carbamoyl phosphate with NarL

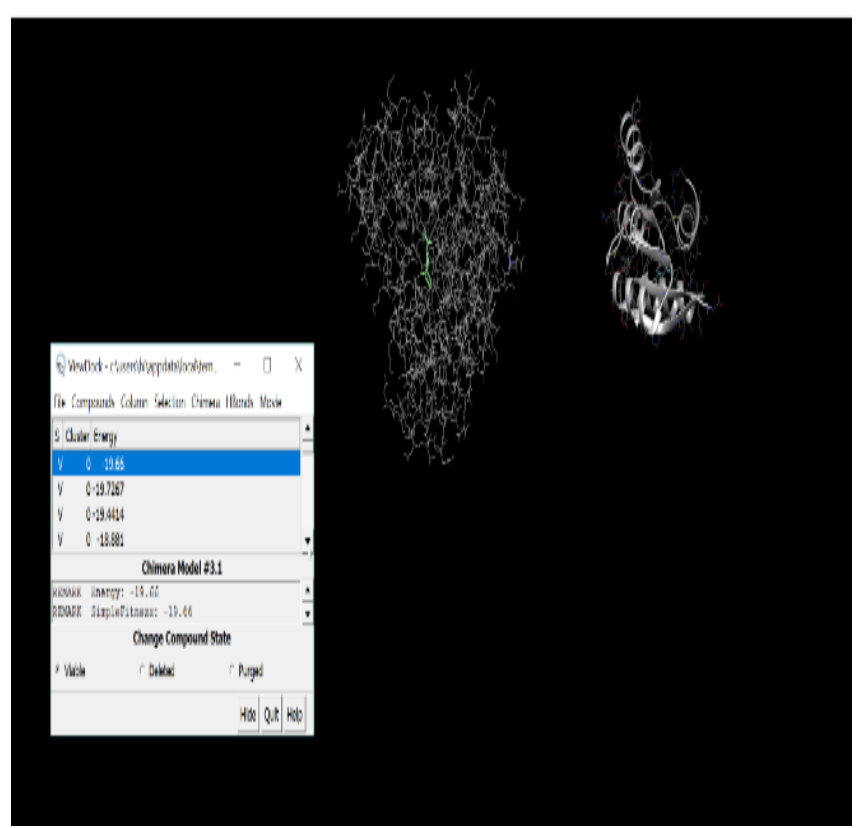

Fig. 4 Docking of Dihydroxyacetone-P with NarL

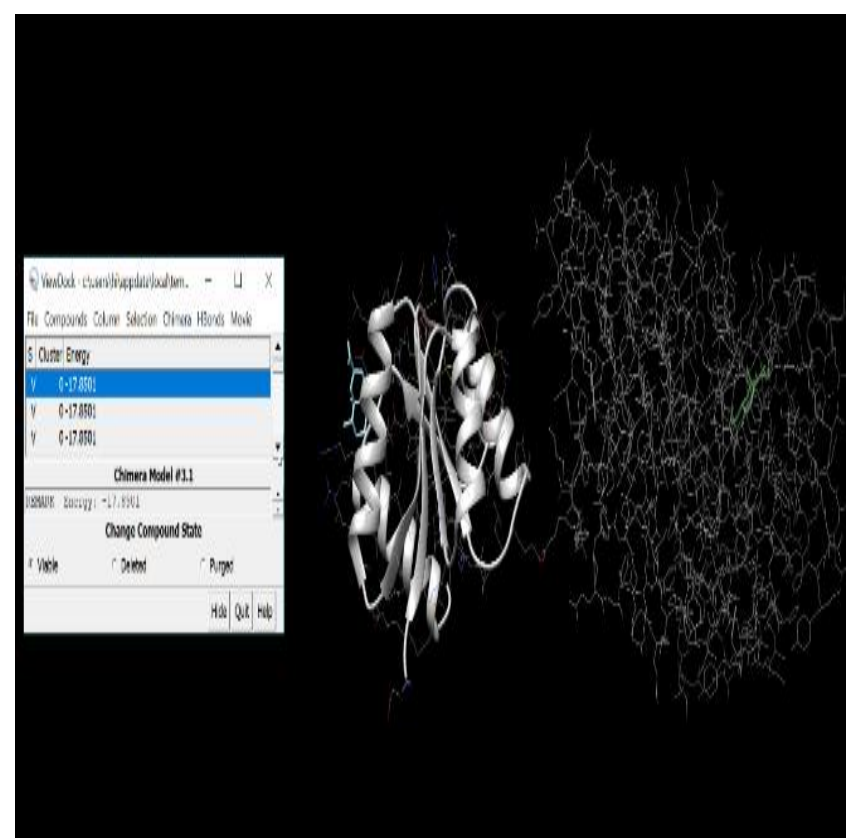

Fig. 5 Docking of Phosphoramidate with NarL

Retrieval Number: B2657129219/2019@BEIESP

DOI: 10.35940/ijeat.B2657.129219

Journal Website: www.ijeat.org

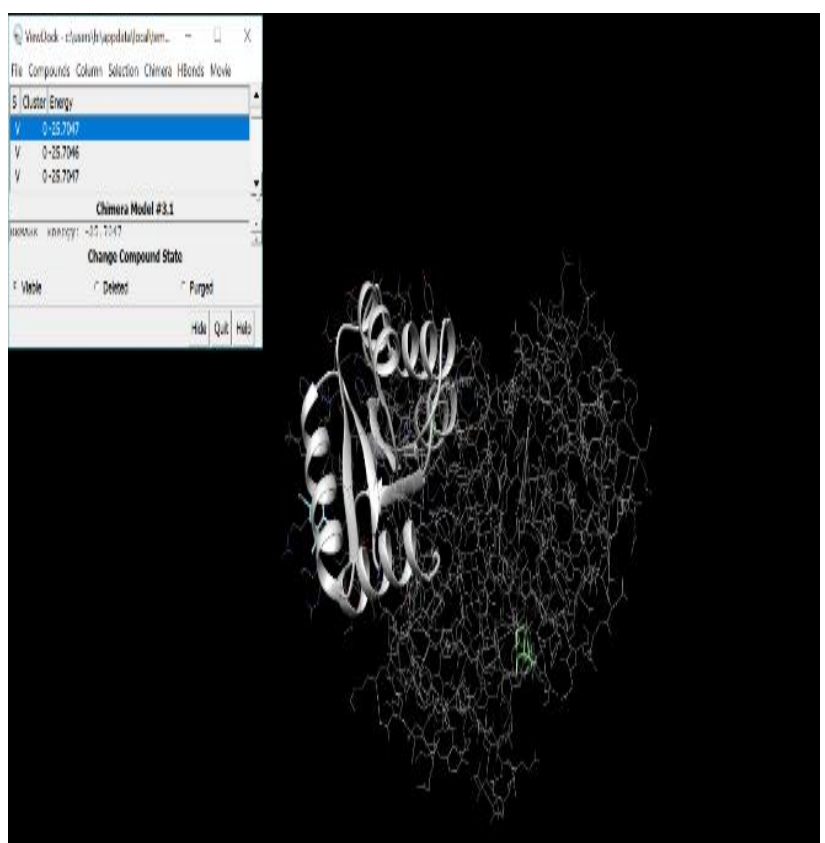

Fig. 6 Docking of Monophosphoimidazole with NarL

\section{CONCLUSION}

The simulation results of molecular docking shows that all compounds are binding to active site, therefore it can be concluded that all the benzofuran and naphthofuran byproducts partake on the dynamic site of NarL and they are able to perform as principal molecule.

\section{ACKNOWLEDGMENT}

This research is funded by the Deanship of Scientific Research at Princess Nourah Bint Abdulrahman University through the Fast-track Research Funding Program.

\section{REFERENCES}

1. Global Tuberculosis Report 2019. Geneva: World Health Organization; 2019. Licence: CC BY-NC-SA 3.0 IGO. ISBN 978-92-4-156571-4.

2. Vinícius de S. Pinto,1 Janay S. C. Araújo,1 Rai C. Silva,2 Glauber V. da Costa,3 Jorddy N. Cruz,4 Moysés F. De A. Neto,5 Joaquín M. Campos,6 Cleydson B. R. Santos, Franco H. A. Leite, and Manoelito C. S. Junior, "In Silico Study to Identify New Antituberculosis Molecules from Natural Sources by Hierarchical Virtual Screening and Molecular Dynamics Simulations”, Pharmaceuticals (Basel). 2019 Mar; Vol.12(1): 36 doi: 10.3390/ph12010036.

3. Prashantha Karunakar, Chamarahalli Ramakrishnaiyer Girija, Venkatappa Krishnamurthy, Venkatarangaiah Krishna, and Kunigal Venugopal Shivakumar, "In Silico Antitubercular Activity Analysis of Benzofuran and Naphthofuran Derivatives." Tuberculosis Research \& Treatment, Hindawi Publishing Corporation Volume 2014, Article ID 697532 pp 1-10 http://dx.doi.org/10.1155/2014/697532

4. Aurélien Grosdidier, Vincent Zoete, Olivier Michielin "SwissDock, a protein-small molecule docking web service based on EADock DSS" Nucleic Acids Research. 2011 Jul 1; doi: 10.1093/nar/gkr366

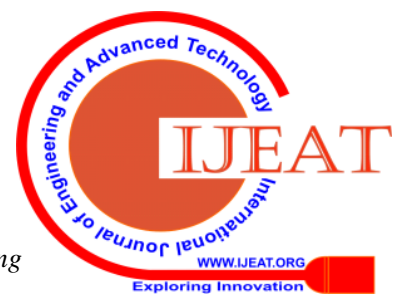




\section{AUTHORS PROFILE}

Shabana Urooj received her B.E. degree in Electrical Engineering and the $\mathrm{M}$. Tech. degree in Electrical with the specialization in Instrumentation and Control from Aligarh Muslim University, Aligarh, India. She completed her Ph.D. in from the Department of Electrical Engineering, Jamia Millia Islamia (A Central University), New Delhi, India. Presently, she is working as Associate Professor at Princess Nourah Bint Abdulrahman University, Riyadh, Kingdom of Saudi Arabia (On leave from Gautam Buddha University, Greater Noida, UP India).

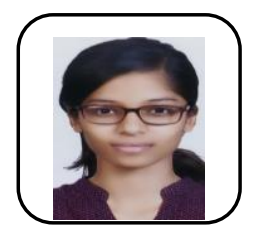

Advaita Dhariwal received her Integrated B.Tech. + M. Tech. Degrees Electrical Engineering and specialization in Instrumentation and Control from Gautam Buddha University, Greater Noida UP India.

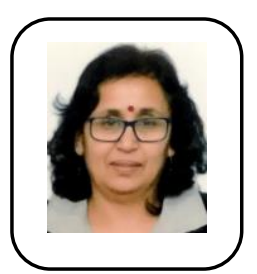

Vandna Singh received her bachelor's and master's degree in chemistry from ccs university meerut. She obtained her doctorate from national institute of technology, kurukshetra haryana, india. She has served as head of the department and various administrative positions. Presently she is a faculty member at school of vocational studies \& applies science, gautam buddha university, gr. Noida up india.

Fadwa M. Alrowais completed her Bachelors and Master's degree in Computer and Information Sciences from King Saud University, Saudi Arabia. She obtained her Ph. D from Faculty of Electronics and Computer Sciences at Southampton University in the United Kingdom. Presently she is heading department of Computer Sciences, College of Computer \& Information Sciences and various other significant administrative and scientific positions at University level. at Princess Nourah Bint Abdulrahman University, Saudi Arabia. 